\title{
The enhanced binding energy for biexcitons in InAs quantum dots
}

\author{
Yasuaki Masumoto, ${ }^{1, a)}$ Seitaro Yoshida, ${ }^{1}$ Michio Ikezawa, ${ }^{1}$ Shinichi Tomimoto, ${ }^{1}$ and \\ Yoshiki Sakuma ${ }^{2}$ \\ ${ }^{1}$ Institute of Physics, University of Tsukuba, Tsukuba 305-8571, Japan \\ ${ }^{2}$ Quantum Dot Research Center, National Institute for Materials Science, Tsukuba 305-0047, Japan
}

(Received 27 September 2010; accepted 22 January 2011; published online 9 February 2011)

\begin{abstract}
We observed that the biexciton binding energy in InAs quantum rhombic disks (QRDs) is enhanced by twice compared with that for InAs quantum dots (QDs) so far reported around $1.24 \mu \mathrm{m}$ nearby the telecommunication wavelength. The heterodyne-detected four-wave-mixing detected the exciton-biexciton quantum beat superposed on photon echo decay, giving the biexciton binding energy of $3.4 \mathrm{meV}$ to 3 monolayer (ML) InAs QRDs and $4.1 \mathrm{meV}$ to $4 \mathrm{ML}$ InAs QRDs, respectively. The largest biexciton binding energy of $4.1 \mathrm{meV}$ in InAs QDs is ascribed to increased electron-hole overlap in confined geometry with a minimized strain distribution. (c) 2011 American Institute of Physics. [doi:10.1063/1.3554425]
\end{abstract}

Biexcitons in quantum dots (QDs) are key elements to realize entangled two-photon sources. Excitons and biexcitons in InAs QDs are especially important because they can cover the most useful wavelength ranging from 1.3 to $1.55 \mu \mathrm{m}$ for optical networks. The binding energy for biexcitons gives the upper limit of temperature above which the biexcitons are unstable. Large biexciton binding energy is required for the stable biexcitons at the elevated temperatures. Although it simply increases with the decrease in the size in nonstrained spherical QDs, ${ }^{1}$ it sensitively depends on shape and size of the strained QDs such as InAs QDs. In fact, biexcitons in InAs QDs have either positive or negative binding energy depending on the strain, shape, and size of them. ${ }^{2-4}$ The general strategy to increase the biexciton binding energy is to increase the overlap of electrons and holes confined in QDs, but the actual one is not clear for InAs QDs. In this report we study biexciton binding energy in InAs quantum rhombic disks (QRDs) in the near infrared region by means of transient four-wave-mixing. The observed exciton-biexciton quantum beat gives the average of biexciton binding energy of an ensemble of QRDs.

By the metal organic chemical vapor deposition, InAs QRDs studied in this work were grown in InP barrier layers on a (001) InP substrate. ${ }^{5}$ On a 2 monolayer (ML) wetting layer $0.6 \mathrm{~nm}$ thick, $1 \mathrm{ML}, 2 \mathrm{ML}$, and $3 \mathrm{ML}$ ultrathin InAs disks are formed by the "double cap method" and are regarded as 3, 4, and 5 ML quantum disks, respectively, as are schematically drawn in the inset of Fig. 1(b). Cross-sectional transmission electron microscopy (TEM) and scanning tunneling microscopy (STM) show extremely flat interface between InAs and InP. ${ }^{5,6}$ Each quantum disk has a rhomb shape whose longer diagonal is $40-50 \mathrm{~nm}$ in the $[1 \overline{1} 0]$ direction and shorter diagonal is $30-40 \mathrm{~nm}$ in the [110] direction, as observed in a plan-view TEM image. The 3 and 4 ML QRDs have a high aspect ratio of $\sim 40$. The areal density of all the disks was $2 \times 10^{10} / \mathrm{cm}^{2}$.

The sample was directly immersed in superfluid helium and was kept at $2 \mathrm{~K}$. An optical parametric oscillator was synchronously pumped by a femtosecond Ti:sapphire laser. It generated 120 fs infrared pulses ranging from 1050 to 1300

${ }^{\text {a)} E l e c t r o n i c ~ a d d r e s s e s: ~ s h o i c h i @ s a k u r a . c c . t s u k u b a . a c . j p . ~}$ $\mathrm{nm}$ at the repetition rate of $81.7 \mathrm{MHz}$. The laser beam was divided into three beams, and optical frequencies of two of them were shifted from $\nu$ into $\nu+80.0 \mathrm{MHz}$ and $\nu$ $+79.0 \mathrm{MHz}$ by two $\mathrm{TeO}_{2}$ acousto-optic modulators operating at 80.0 and $79.0 \mathrm{MHz}$, respectively. Resonant pulses at the optical frequencies $\nu+80.0 \mathrm{MHz}$ and $\nu$ excited the sample coaxially in the Brewster-angle reflection configuration. The polarization of both the excitation beams was parallel to the [110] axis of the crystal. The four-wave-mixing signal was mixed with the laser beam at $\nu+79.0 \mathrm{MHz}$, and the mixed signal modulating at $81.0 \mathrm{MHz}$ was detected by a

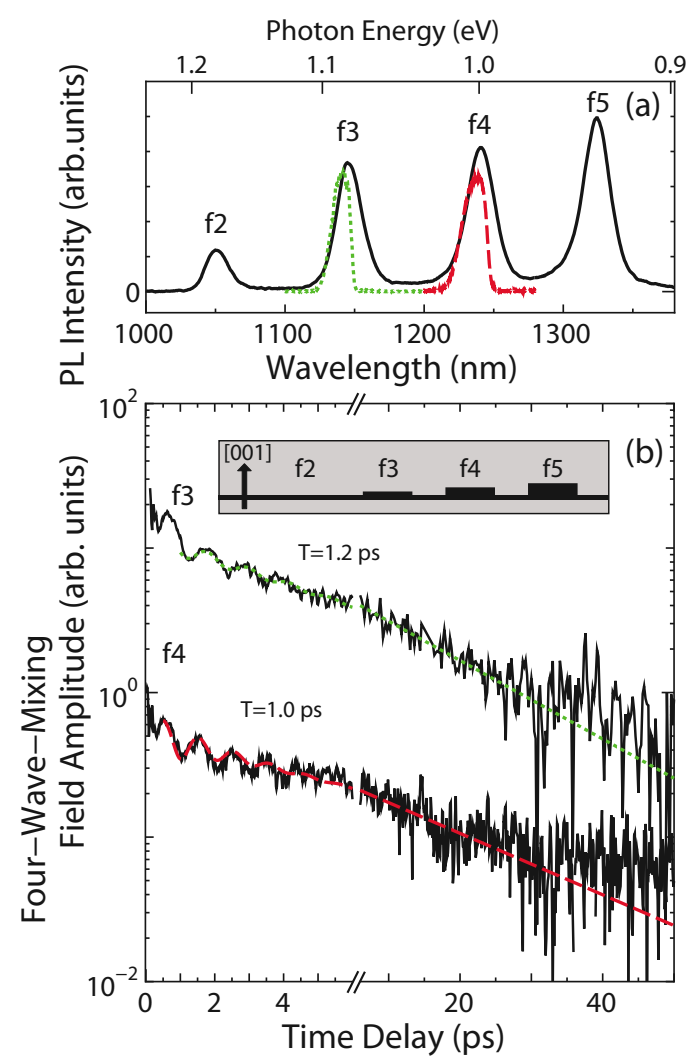

FIG. 1. (Color online) (a) PL spectrum of InAs quantum structures and laser spectrum used for the four-wave-mixing experiment. (b) Temporal change of four-wave-mixing field amplitude emitted from InAs QRDs. A wetting layer and QRDs are schematically shown in the inset. 


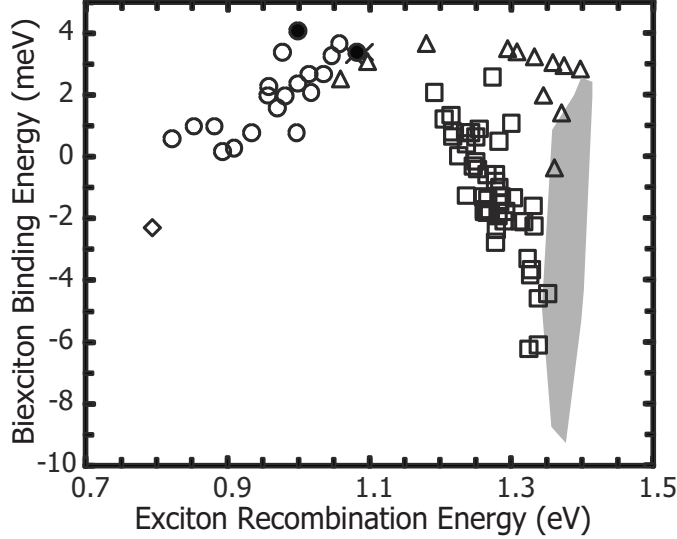

FIG. 2. Biexciton binding energies observed in InAs/InP QRDs (-) and those observed in InAs/InP QDs ( $\bigcirc$; see Refs. 3, 11, and 12), InAs/InGaAs QDs ( $\times$; see Ref. 13), InAs/InGaAsP QDs $(\diamond$; see Ref. 14$)$, InAs/GaAs QDs ( $\square$; see Refs. 2 and 15), InAs/GaAs QDs (shade; see Ref. 4), and InGaAs/GaAs QDs $(\triangle$; see Refs. 16-18).

balanced high-frequency InGaAs pin photodiode and a spectrum analyzer. Further the signal was amplified by a lock-in amplifier at the mechanical chopping frequency of $90 \mathrm{~Hz}$. The highly sensitive infrared heterodyne detection allowed us to observe the four-wave-mixing signal from a single layer of InAs QRDs. ${ }^{7,8}$

The photoluminescence (PL) spectrum of the sample is shown in Fig. 1(a). It is composed of bands peaked at $1052 \mathrm{~nm}(1.179 \mathrm{eV}), 1142 \mathrm{~nm}(1.082 \mathrm{eV}), 1238 \mathrm{~nm}(0.999$ $\mathrm{eV})$, and $1310 \mathrm{~nm}(0.937 \mathrm{eV})$ assigned to come from a $2 \mathrm{ML}$ InAs quantum well corresponding to a wetting layer (f2), 3 ML InAs disks (f3), 4 ML disks (f4), and 5 ML disks (f5), respectively. When the $\mathrm{f} 3$ band is excited resonantly by two linearly polarized pulses, we observed a four-wavemixing signal shown in Fig. 1(b). It is composed of a quantum beat and a photon echo decay showing the dephasing time of excitons and is fitted by the expression $A \cos (2 \pi t / T) \exp \left(-t / \tau^{\prime}\right)+B \exp (-t / \tau)$. The decay time $\tau$ of the echo field amplitude is $16 \mathrm{ps}$ and the dephasing time of the excitons in $3 \mathrm{ML} \mathrm{QRDs}$ is $T_{2}=2 \tau=31 \mathrm{ps}$. The dephasing time is much shorter than the radiative lifetime of the excitons in $3 \mathrm{ML}$ QRDs, $1.58 \mathrm{~ns}$. ${ }^{9}$ The quantum beat starts with an oscillation minimum at $t=0$ and oscillates with a period of $T=1.2 \mathrm{ps}$, reflecting the biexciton binding energy defined by the energy difference between exciton transition and excitonbiexciton transition. The oscillation minimum at $t=0$ shows the exciton-biexciton quantum beat. ${ }^{10}$ The biexciton binding energy of $3.4 \mathrm{meV}$ was obtained. When the $\mathrm{f} 4$ band is excited resonantly, the decay time of the photon echo is prolonged and the dephasing time is $T_{2}=41 \mathrm{ps}$. The excitonbiexciton quantum beat is superposed on the photon echo, and the beat period of $T=1.0$ ps gives the biexciton binding energy of $4.1 \mathrm{meV}$. The exponential damping time of the beat, $\tau^{\prime}$, shows the biexciton dephasing time of 1.8 ps for 3 ML QRDs and 1.3 ps for 4 ML QRDs. Comparing biexciton binding energies of 3.4 and $4.1 \mathrm{meV}$, we note that biexciton binding energy increases with the increase of height of QRDs.

Biexciton binding energies of 3.4 and $4.1 \mathrm{meV}$ obtained in the present work are plotted as a function of the exciton recombination energy in Fig. 2. So far biexciton binding energies for InAs/InP QDs, ${ }^{3,11}$ In Ins/InGaAs QDs, ${ }^{13}$ InAs/ InGaAsP QDs, ${ }^{14}$ InAs/GaAs QDs, ${ }^{2,4,15}$ and InGaAs/GaAs
QDs (Refs. 16-18) have been reported. They are also plotted in Fig. 2. Looking at Fig. 2, we found that $4.1 \mathrm{meV}$ is the largest biexciton binding energy in InAs QDs. Moreover 4.1 $\mathrm{meV}$ is twice of $2.0 \mathrm{meV}, 5$ adjacent average of biexciton binding energies around the exciton recombination energy of $0.999 \mathrm{eV}$ measured by single dot spectroscopy. ${ }^{3}$ It is also noted that the most strained systems, InAs/GaAs QDs, do not have large biexciton binding energies.

Reminding of theoretical and experimental works on biexciton binding energy in spherical QDs, biexciton binding energy increases monotonously with the decrease in the size of QDs in both strong and weak confinement regimes. ${ }^{1}$ However this is not the case for 4 and 3 ML InAs QRDs and InAs QDs reported, ${ }^{2}$ as seen for the exciton recombination energy ranging from 1.0 to $1.26 \mathrm{eV}$ in Fig. 2. On the other hand, biexciton binding energy decreases with the decrease in the exciton recombination energy ranging from 0.9 to $1.0 \mathrm{eV}$ in Fig. 2. Increasing the size reduces the overlap of electrons and holes in the QDs. Further increasing the size increases the strain distribution in the QDs and decreases the biexciton binding energy, because strain field causes piezoelectric field in InAs QDs which spatially separates electrons and holes leading to reduction in the electron-hole attractive Coulomb energy and exchange energy and increase in electronelectron and hole-hole repulsive Coulomb energy.

To explain the anticorrelation of biexciton binding energy and the exciton transition energy observed for $4 \mathrm{ML}$ InAs QRDs and $3 \mathrm{ML}$ InAs QRDs, we remind that the radiative lifetime of $4 \mathrm{ML}$ InAs QRDs is $\tau_{r}=1.4 \mathrm{~ns}$ and is shorter than that of $3 \mathrm{ML}$ InAs QRDs, $\tau_{r}=1.6 \mathrm{~ns}$, by $14 \%$. $^{9}$ The radiative rate $1 / \tau_{r}$ of QDs is proportional to $f \omega^{2}$, where $f$ is the oscillator strength and $\omega$ is the optical transition frequency. Using the optical transition energies of $1.00 \mathrm{eV}$ for $\mathrm{f} 4$ and $1.08 \mathrm{eV}$ for $\mathrm{f} 3$, we can evaluate that $f$ for $4 \mathrm{ML}$ InAs QRDs is larger than $f$ for 3 ML InAs QRDs by $30 \%$. The disk-height dependence of $f$ gives the overlap of the electron-hole wave function. The effective mass of the electron is smaller than that of the hole by an order of magnitude, and the conduction band discontinuity is reduced by the strain, as discussed later. As a result, electron wave function spills from the $3 \mathrm{ML}$ InAs QRDs more than from the $4 \mathrm{ML}$ InAs QRDs, while the hole is well confined in the quantum disks. The spillover of the electron wave function reduces the electron-hole attractive Coulomb energy and exchange energy and hence reduces the biexciton binding energy.

Minimizing the strain distribution in QDs is also important to increase the biexciton binding energy. Lattice constants of freestanding zinc-blende InAs and InP are $a(\operatorname{InAs})$ $=0.608 \mathrm{~nm}$ and $a(\mathrm{InP})=0.587 \mathrm{~nm}$, respectively. Heterointerface (001) between InAs and InP has a lattice mismatch of $3.45 \%$. Because the $2 \mathrm{ML}$ thick InAs wetting layer is much thinner than InP layers and is within the critical thickness for the onset of strain relaxation, ${ }^{19}$ the InAs layer alone is coherently strained. In fact, dark shade reflecting the strain field is absent at both sides of the InAs wetting layer, as seen in the cross-sectional TEM. ${ }^{5}$ Although biaxial compressive strain $\epsilon_{\text {InAs } \|}=-0.0345$ is applied to a thin InAs layer along the interface, uniaxial tensile strain $\epsilon_{\mathrm{InAs} \perp}=0.0376$ is perpendicular to the interface, and the InAs layer is elongated in the [001] direction. According to the model-solid theory, ${ }^{20}$ the 
coherent strain increases the valence band discontinuity to $\Delta E_{v}=0.40 \mathrm{eV}$, while decreases the conduction band discontinuity to $\Delta E_{c}=0.39 \mathrm{eV}$. Electron wave function spills from the 2 ML InAs quantum well, while the hole is well confined in the quantum well, because the effective mass of the electron is smaller than that of the hole by an order of magnitude. ${ }^{21}$ Coherent strain reduces the biexciton binding energy only through the reduction of the symmetric electronhole overlap.

On the other hand, dark shade seen just above the interface in the cross-sectional TEM (Ref. 5) shows that biaxial tensile strain is present in an InP upper layer. It means that biaxial compressive strain is relaxed somewhat at the upper InAs/InP interface of QRDs. On the contrary, dark shade seen outside the interface in the plan-view TEM (Ref. 5) shows that uniaxial tensile strain is applied in the [001] direction nearby the edge of InAs rhomb in the InP layer. It means that uniaxial compressive strain is applied in the [001] direction in the InAs QRDs, but strain itself is weakened at the upper InAs/InP interface of QRDs because of their large aspect ratio. Further intermixing between As and $\mathrm{P}$ seen in the cross-sectional STM is considered to reduce the strain field considerably. In fact, the strain field applied in the InAs QRDs is much weaker than the InAs QDs, because dark shade observed for InAs QRDs in the cross-sectional TEM (Ref. 5) is lighter and more limited spatially than that observed for InAs QDs grown by the "normal-capping procedure." A large aspect ratio reduces the strain distribution in InAs QRDs. It prevents the asymmetric electron-hole separation and the decrease in biexciton binding energy. We consider that $4 \mathrm{ML}$ InAs/InP QRDs have optimum size and shape for the large biexciton binding energy for InAs QDs.

In summary, we observed the largest biexciton binding energy in InAs QDs through exciton-biexciton quantum beat by means of the highly sensitive heterodyne-detected fourwave-mixing. It is $4.1 \mathrm{meV}$ for $4 \mathrm{ML}$ InAs QRDs and twice of the biexciton binding energy in InAs QDs so far reported at $1.24 \mu \mathrm{m}$ nearby the telecommunication wavelength. A strategy to increase the biexciton binding energy in InAs QDs is to reduce the strain distribution in the quantum disks with a high aspect ratio as well as spillover of the electron wave function from the thin quantum disks.
This work was supported by Grant-in-Aid for Scientific Research No. 20244044 from the MEXT of Japan.

${ }^{1}$ A. D. Yoffe, Adv. Phys. 50, 1 (2001).

${ }^{2}$ S. Rodt, R. Heitz, A. Schliwa, R. L. Sellin, F. Guffarth, and D. Bimberg, Phys. Rev. B 68, 035331 (2003).

${ }^{3}$ N. Chauvin, B. Salem, G. Bremond, G. Guillot, and C. Bru-Chevallier, J. Appl. Phys. 100, 073702 (2006).

${ }^{4}$ R. J. Young, R. M. Stevenson, A. J. Shields, P. Atkinson, K. Cooper, D. A. Ritchie, K. M. Groom, A. I. Tartakovskii, and M. S. Skolnick, Phys. Rev. B 72, 113305 (2005); many data points in this paper are in the shaded zone in Fig. 2.

${ }^{5}$ Y. Sakuma, M. Takeguchi, K. Takemoto, S. Hirose, T. Usuki, and N. Yokoyama, J. Vac. Sci. Technol. B 23, 1741 (2005).

${ }^{6}$ Y. Akanuma, I. Yamakawa, Y. Sakuma, T. Usuki, and A. Nakamura, Appl. Phys. Lett. 90, 093112 (2007).

${ }^{7}$ Y. Masumoto, F. Suto, M. Ikezawa, C. Uchiyama, and M. Aihara, J. Phys. Soc. Jpn. 74, 2933 (2005).

${ }^{8}$ M. Ikezawa, S. V. Nair, H.-W. Ren, Y. Masumoto, and H. Ruda, Phys. Rev. B 73, 125321 (2006).

${ }^{9}$ S. Tomimoto, A. Kurokawa, Y. Sakuma, T. Usuki, and Y. Masumoto, Phys. Rev. B 76, 205317 (2007).

${ }^{10}$ T. F. Albrecht, K. Bott, T. Meier, A. Schulze, M. Koch, S. T. Cundiff, J. Feldmann, W. Stolz, P. Thomas, S. W. Koch, and E. O. Göbel, Phys. Rev. B 54, 4436 (1996).

${ }^{11}$ D. Chithrani, R. L. Williams, J. Lefebvre, P. J. Poole, and G. C. Aers, Appl. Phys. Lett. 84, 978 (2004).

${ }^{12}$ G. Saint-Girons, N. Chauvin, A. Michon, G. Patriarche, G. Beaudoin, G. Brémond, C. Bru-Chevallier, and I. Sagnes, Appl. Phys. Lett. 88, 133101 (2006).

${ }^{13}$ S. Kaiser, T. Mensing, L. Worschech, F. Klopf, J. P. Reithmaier, and A. Forchel, Appl. Phys. Lett. 81, 4898 (2002).

${ }^{14}$ N. I. Cade, H. Gotoh, H. Kamada, H. Nakano, S. Anantathanasarn, and R. Nötzel, Appl. Phys. Lett. 89, 181113 (2006).

${ }^{15}$ R. Seguin, A. Schliwa, T. D. Germann, S. Rodt, K. Pötschke, A. Strittmatter, U. W. Pohl, D. Bimberg, M. Winkelnkemper, T. Hammerschmidt, and P. Kratzer, Appl. Phys. Lett. 89, 263109 (2006).

${ }^{16}$ J. J. Finley, A. D. Ashmore, A. Lemaître, D. J. Mowbray, M. S. Skolnick, I. E. Itskevich, P. A. Maksym, M. Hopkinson, and T. F. Krauss, Phys. Rev. B 63, 073307 (2001).

${ }^{17}$ W. Langbein, P. Borri, U. Woggon, V. Stavarache, D. Reuter, and A. D. Wieck, Phys. Rev. B 69, 161301(R) (2004).

${ }^{18}$ F. Ding, R. Singh, J. D. Plumhof, T. Zander, V. Krápek, Y. H. Chen, M. Benyoucef, V. Zwiller, K. Dörr, G. Bester, A. Rastelli, and O. G. Schmidt, Phys. Rev. Lett. 104, 067405 (2010).

${ }^{19}$ J. Cibert, Y. Gobil, L. S. Dang, S. Tatarenko, G. Feuillet, P. H. Jouneau, and K. Saminadayar, Appl. Phys. Lett. 56, 292 (1990).

${ }^{20}$ C. G. Van de Walle, Phys. Rev. B 39, 1871 (1989).

${ }^{21}$ U. Cebulla, G. Bacher, A. Forchel, G. Mayer, and W. T. Tsang, Phys. Rev. B 39, 6257 (1989). 